\title{
A DYNAMIC TECHNIQUE FOR MEASURING SURFACE TENSION AT HIGH TEMPERATURES IN MICROGRAVITY ENVIRONMENT
}

\author{
A.P. Miiller and A. Cezairliyan \\ Thermophysics Division \\ National Institute of Standards and Technology \\ Gaithersburg, MD 20899
}

\begin{abstract}
The feasibility of a dynamic technique for measuring surface tension of liquid metals at high temperatures in a microgravity environment has been demonstrated. The basic method involves heating a tubular specimen resistively from ambient temperature through its melting point in about $1 \mathrm{~s}$ by passing an electrical current pulse through it, while simultaneously recording the pertinent experimental quantities. Static equilibrium for the molten specimen is achieved in a microgravity environment by splitting the current after it passes through the specimen tube and returning a fraction along the tube axis, and the remaining fraction outside the specimen. Adjustments to the current split enable a balance between the magnetic and surface tension forces acting on the specimen. Values for surface tension are determined from measurements of the equilibrium dimensions of the molten specimen tube, and the magnitudes of the currents. Rapid melting experiments, performed during microgravity simulations with NASA's KC-135 aircraft, yield a value for the surface tension of copper at its melting point which is in agreement with literature dala. Measurements of surface tension of a refractory metal (tantalum) are underway.
\end{abstract}




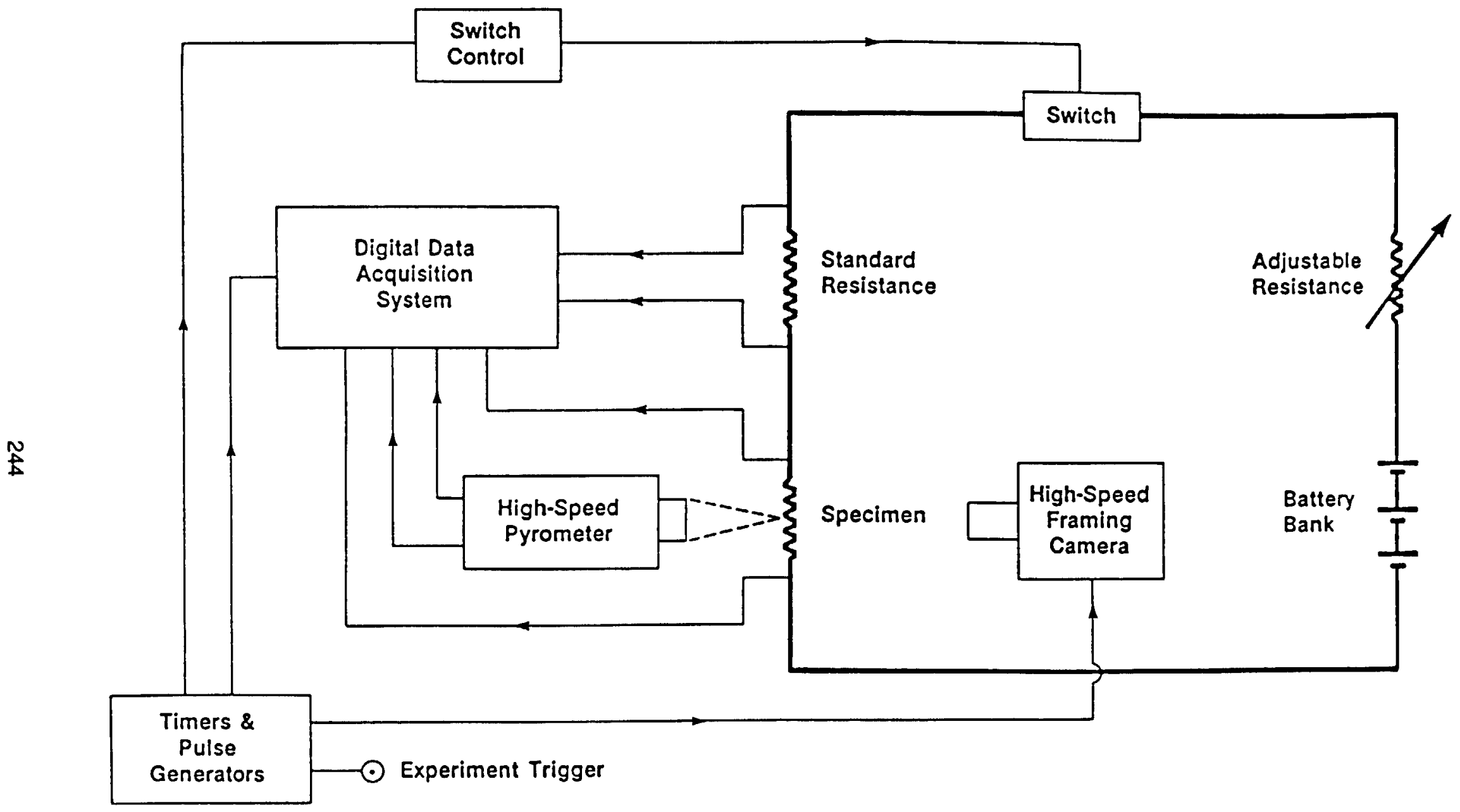

Fig. 1. A functional diagram of a compact pulse-heating system designed for rapid-melting experiments during microgravity simulations with the KC-135 aircraft. 


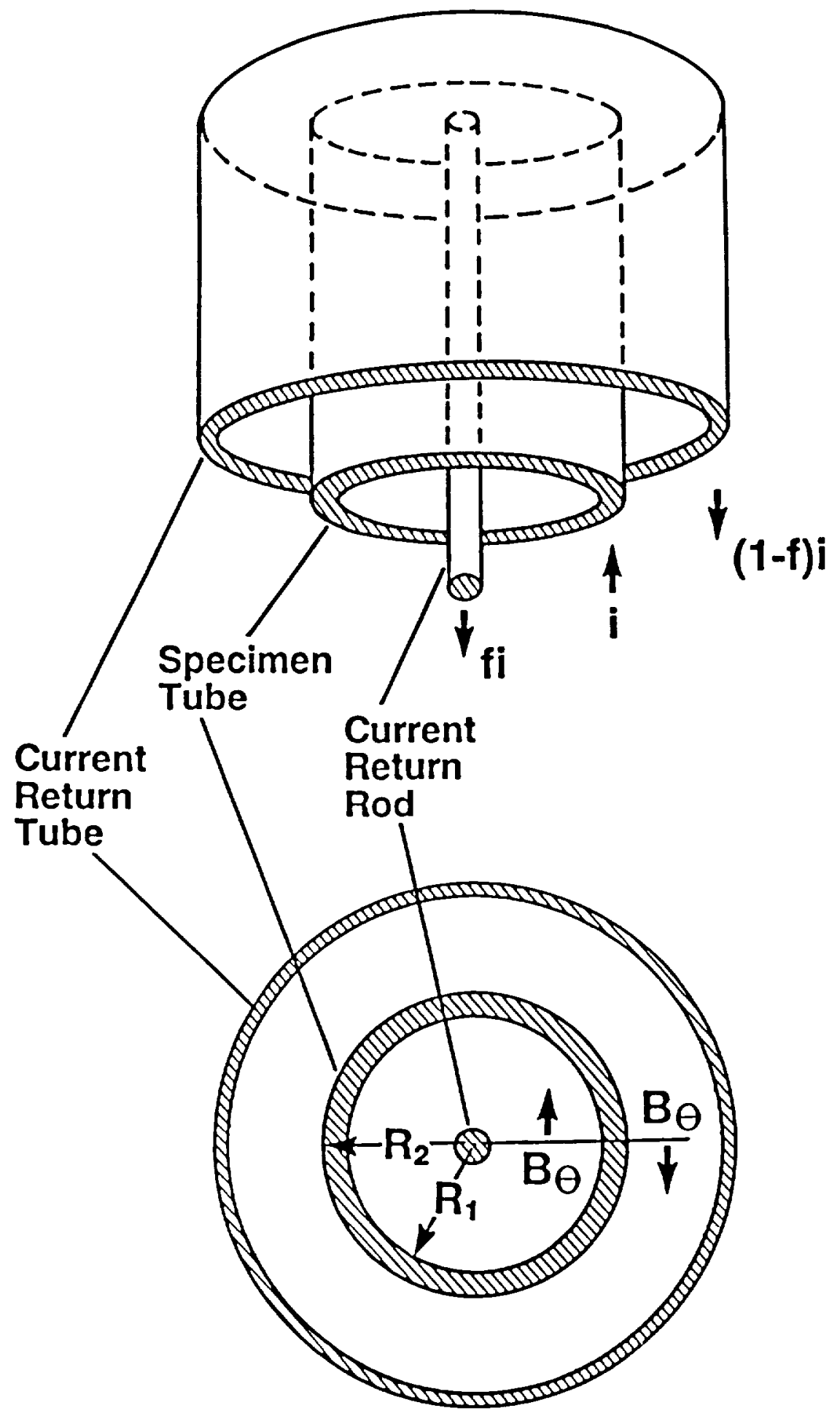

Fig. 2. A schematic diagram of the triaxial configuration in which a tubular specimen in mounted concentrically with respect to the current return paths. During melting, the (inward) pressure due to surface tension may be counter-balanced by selecting a suitable return current split $f$ to provide a net (outward) magnetic pressure. 


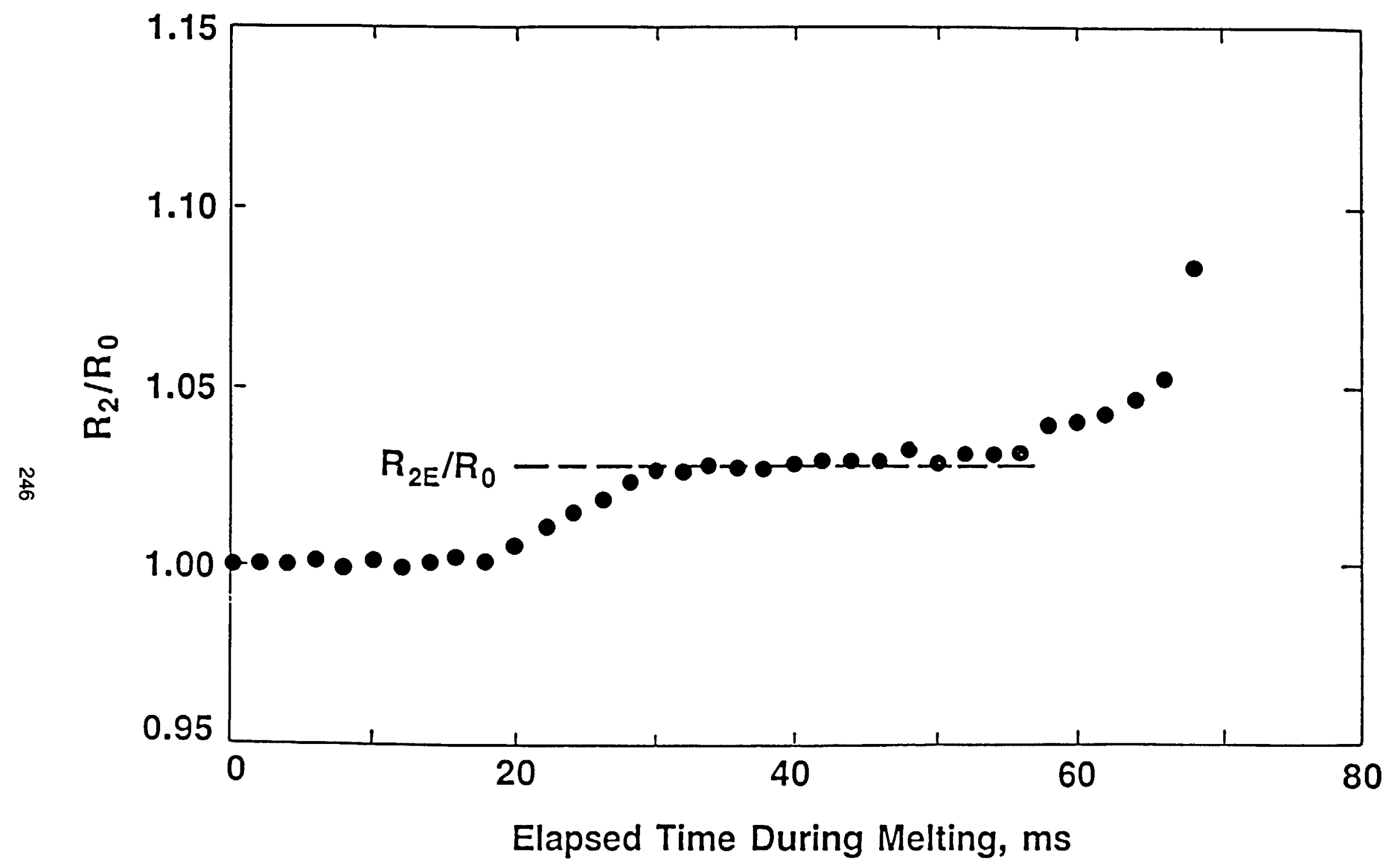

Fig. 3. Variation of the melt zone radius $R_{2}$ of a thin-walled copper tube during rapid melting under microgravity conditions; $R_{0}$ is the tube radius just before melting and $R_{2 E}$ is the tube radius at static equilibrium during melting. 


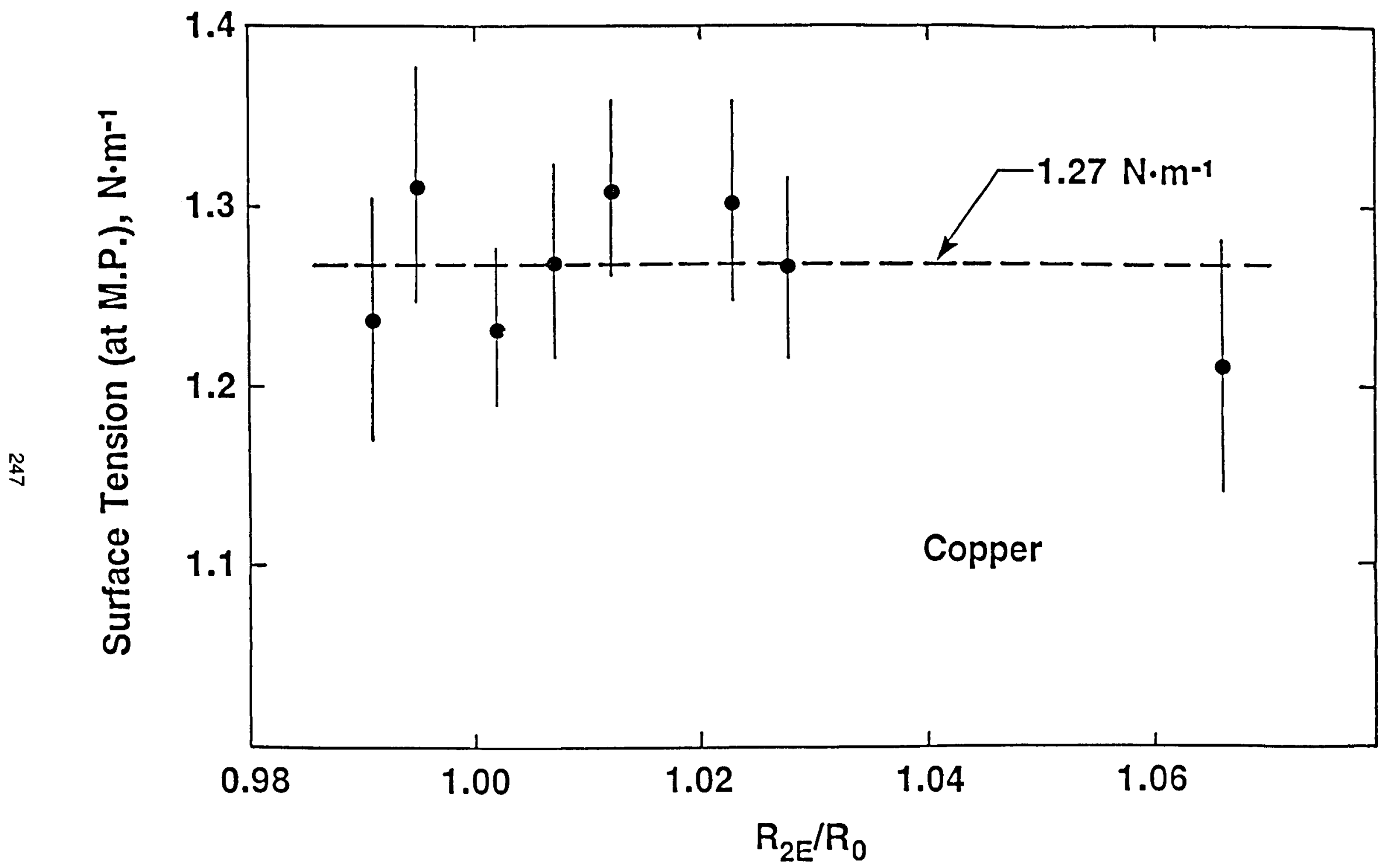

Fig. 4. Results from eight microgravity experiments for surface tension of copper (at its melting point) as determined from measurements of the equilibrium dimensions of the molten tube and the magnitudes of the currents. 